\title{
Disease and Diagnosis
}

\section{Comparing the Effects of Aloe Vera Ointment with Routine Treatment on the Severity of Diaper Dermatitis: A Randomized Controlled Double-Blinded Clinical Trial}

\author{
Shiva Heidari ${ }^{\circledR}$, Naser Parizad $^{2}{ }^{\circledR}$, Fatemeh Shirazi $^{3^{\circledR}}$, Vahid Alinejad $^{4}$, Sima Ghanipour Badelbuu ${ }^{2}$ B $^{\circledR}$ \\ ${ }^{1}$ Nursing and Midwifery School, Urmia Islamic Azad University, Urmia, Iran. \\ ${ }_{2}^{2}$ Patient Safety Research Center, Clinical Research Institute, Nursing \& Midwifery School, Urmia University of Medical \\ Sciences, Urmia, Iran. \\ ${ }^{3}$ Nursing and Midwifery School, Shiraz University of Medical Sciences, Shiraz, Iran. \\ ${ }^{4}$ Medical School, Urmia University of Medical Sciences, Urmia, Iran.
}

\begin{abstract}
Background: Diaper dermatitis (DD) is a prevalent skin disease in children, which causes child discomfort and parental concern. This study aimed to compare the effects of aloe vera ointment with routine treatment on the severity of DD in hospitalized children.

Materials and Methods: This double-blinded randomized clinical trial was carried out on 60 children aged less than two years admitted to Tabriz pediatric hospital in Iran from February to June 2017. Using convenience sampling method, the patients were assigned into two equal groups $(n=30$ in each) of intervention and control. The intervention group received routine combination treatment, whereas the control group received aloe vera ointment. The patients were examined on the first, third, and sixth days of the study. Data analysis was performed using SPSS software (version 22) and descriptive and analytical statistics.

Results: The severity of DD in both groups improved on the sixth day compared to the first day. In comparison, no significant difference was observed between the two groups regarding the severity of dermatitis $(P>0.05)$.

Conclusion: Herbal products cause fewer side effects than chemical products and seem to have significant pharmacological effects on the skin. Thus, aloe vera ointment appears to help treat DD and, with further studies, may emerge as a possible therapeutic option.

Keywords: Diaper rash, Aloe vera, Ointments, Randomized controlled trial, Iran
\end{abstract}

*Correspondence to Sima Ghanipour Badelbuu, Patient Safety Research Center, Clinical Research Institute, Nursing \& Midwifery School, Urmia University of Medical Sciences, Urmia, Iran. Tel: +984432754961 ; Email: ghanipourfrnzvsf@ gmail.com

Received: December 19, 2020, Accepted: December 26, 2020, ePublished: December 30, 2020

\section{Introduction}

Diaper dermatitis (DD), also known as nappy rash and irritant napkin dermatitis, is dermatitis typically limited, at least primarily, to the area covered by a diaper. It is only observed when diapers are used (1). The disease often develops 3-12 weeks after birth, and it usually peaks between 6 and 9 months of age (2). The disorder could eventually lead to bacterial and fungal infections (3). Higher-risk areas for DD include the groin, abdomen, genitals, thighs, gluteal area, and the perianal area, which gradually heal within a few days; otherwise, it should be treated medically (4). Because DD improves spontaneously in most cases, there is no requirement for referrals to medical treatment. Hence, data are not available on the exact prevalence rate of the disease, while some recent studies have reported the rate to be between $25 \%$ to $50 \%$ in children aged $6-12$ months $(4,5)$.

Several therapeutic agents are used to treat DD
(2). A combination of topical medications, such as corticosteroids and antifungals drugs under the barrier creams are usually used to control inflammation and fungal infection in children with severe DD. If a secondary bacterial infection is expected, a topical antibacterial drug may be added to treatment medications (6). Aloe vera has numerous medicinal properties, such as antiinflammatory, antifungal, antibacterial, antiviral, and wound-healing effects, accelerating wound healing (7). These chemical medications, that are routinely used to treat DD, cause numerous complications, such as secondary rashes, telangiectasia, skin atrophy, and allergic dermatitis.

Medicinal plants are frequently used to treat various diseases, including DD $(2,8)$. Herbal remedies do not cause the same adverse effects as chemical medications and are also affordable (9). Some of the most common herbal medicines used for the treatment of DD include

(C) 2020 The Author(s). This is an open access article distributed under the terms of the Creative Commons Attribution License (http:// creativecommons.org/licenses/by/4.0/), which permits unrestricted use, distribution, and reproduction in any medium, provided the original work is properly cited. 
calendula, chamomile, lanolin, and sunflower oil $(2,10,11)$. The use of aloe vera, vitamins $\mathrm{A}$ and $\mathrm{E}$, honey, olive oil, wax, and henna was reported in recent studies $(2,3,12)$. Aloe vera is a medicinal plant with the scientific name Aloe barbadensis (Miller), which belongs to the Liliaceae family, and it has been used as a traditional medicine to treat a verity of illnesses, including skin and gastrointestinal disorders $(3,12)$. Some of the prominent features of this plant include wound healing, antiinflammatory, anti-diabetic, anticancer, antioxidant, and antiulcer effects $(3,7,13)$. Although aloe vera has been shown to have healing effects on various diseases, limited studies have been conducted on the effect of aloe vera ointments on $\operatorname{DD}(3,11)$.

Recent review studies showed the lack of sufficient clinical trials to determine which specific skincare practices are clinically effective in managing DD (2). Thus, conducting further studies on traditional medicines, especially clinical trials, seems essential to fill the existing gaps in current knowledge $(2,7,12)$. Accordingly, the present study aimed to compare the effects of aloe vera ointment with routine treatment on the severity of DD in children.

\section{Materials and Methods \\ Study Design and Population}

This double-blinded, randomized, controlled, clinical trial was conducted on 60 children aged less than two years admitted to Tabriz pediatric hospital in Iran from February to June 2017. The inclusion criteria were as follows: willingness to participate in the study; the age of 0-24 months; using disposable diapers; not having known systemic diseases such as acrodermatitis enteropathica from zinc deficiency, hand-foot-mouth disease, psoriasis, and cutaneous candidiasis (14); and not using systemic medications. The exclusion criteria included unwillingness to participate in the study; discharging home; having fungal infections; using reusable cloth diapers; having positive stool culture; and allergies to treatment ointments.

The research team considered the severity of dermatitis as the primary outcome in this study. The sample size was determined based on the previous study (11). Based on $a=0.05$, power of $80 \%$, and $10 \%$ reduction difference between the two groups, 56 samples were calculated using $\mathrm{G}^{\star}$ Power software. Finally, considering $10 \%$ probability of attrition rate, 60 samples entered the study. The patients who met inclusion criteria were selected by convenience sampling method, and allocated into aloe vera $(n=30)$ and routine treatment $(n=30)$ groups using the Random List Generator Software (Figure 1). Two patients in the control group were discharged to home, and excluded from the study.

\section{Preparation of Ointments}

The study ointments included 95\% aloe vera ointment and routine treatment ointment. The ointments prepared by a pharmacist in Tabriz School of Pharmacy in Iran were presented in white tubes with similar shape and size, and both the researchers and mothers were blinded to the type of ointment. To prepare 95\% Aloe vera ointment, the pharmacist first soaked the aloe vera plant in $70 \%$ hydro-alcoholic solution and then placed it on a shaker for 72 hours to obtain the whole extract. The solution was filtered off and placed in a rotary evaporator to remove the solvent. The obtained powder was grounded and kept away from light and moisture in the refrigerator until the ointment was ready. An appropriate amount of dry plant extract with preservatives, such as methylparaben and propylparaben was added to the Eucerin base to prepare the ointments, and geometrically stirred to create a uniform mixture. The aloe vera and routine ointments were filled and packed in similar $50 \mathrm{~g}$ tubes.

\section{Intervention}

After selecting the patients and obtaining parental consent to participate in the study, the parents were examined for skin allergies $\left(1^{\star} 1 \mathrm{~cm}\right)$ with ointment and checked after 20 minutes. The aloe vera ointment (A) was applied to children in the intervention group, and routine treatment ointment (B) was used in the involved area of children in the control group. The routine treatment (B) composed of a combination of hydrocortisone, clotrimazole, and zinc

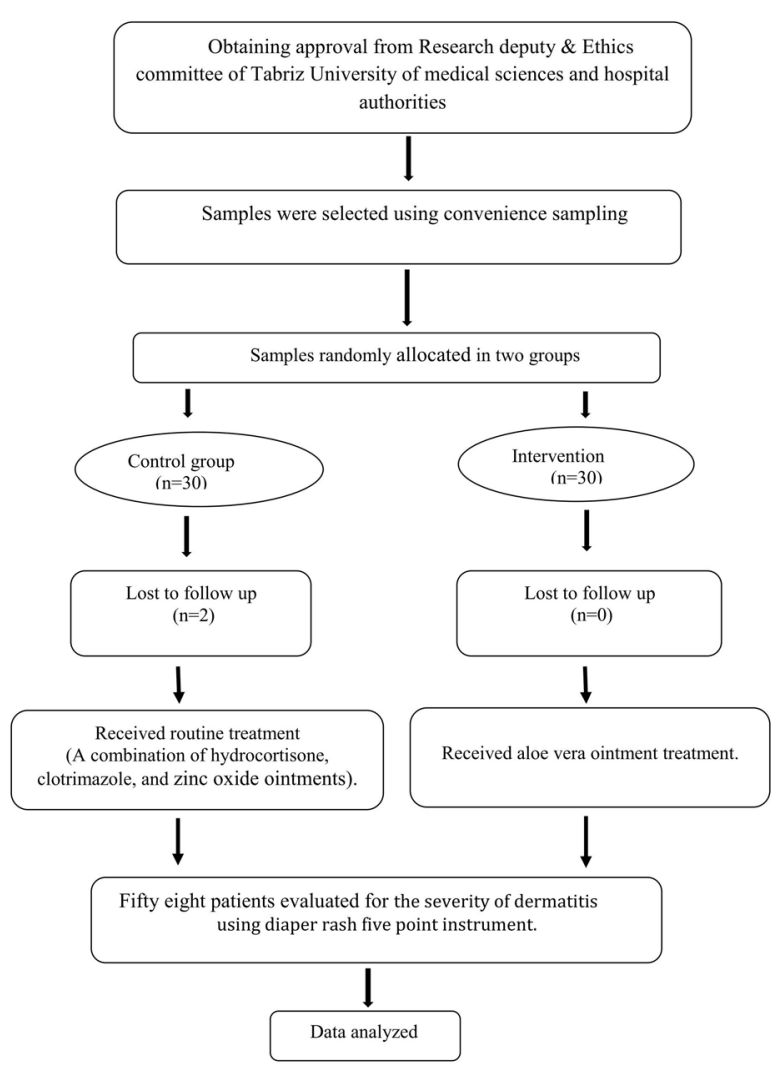

Figure 1. The Flow Diagram of the Study Based on Consort Statement 2012, Indicating Participants' Recruitment and Intervention Process 
oxide ointments (6). In both groups, the mothers of the children were instructed to apply a layer of the ointment three times a day after washing or removing all residual from the previous dose of ointment gently with lukewarm water and mild soap to cover the lesion completely. Afterward, the severity of dermatitis was determined on the first, third, and sixth days of the study by a trained researcher.

\section{Instruments}

For measuring the severity of dermatitis, a five-point diaper rash instrument was used based on a study by Al-Waili conducted in the United States (15). Fifty-eight patients were evaluated by a trained researcher who was blind to the groups. In this instrument, the severity of $\mathrm{DD}$ with the score 0 interpreted as no erythema, score 1 defined as mild erythema, score 2 interpreted as moderate erythema, score 3 defined as severe erythema with swelling, and score 4 showing severe erythema with swelling and ulcer. Noonan et al confirmed the interrater agreement, and two observers reached $90 \%$ agreement in their study (16). The agreement coefficient of observers in the study by Afshari et al was calculated to be 0.8 (17). The interrater reliability of the instrument was rechecked and confirmed in this study. We used the observer agreement coefficient to determine the reliability of the instrument. Two observers determined the severity of the first ten cases of DD, and the degree of agreement of the observers in this study was 0.83 .

\section{Data Analysis}

Data were analyzed in IBM SPSS Statistics version 22.0 using independent $t$ test and chi-square at the significance level of 0.05 and $0.95 \%$ confidence coefficient. Two patients were discharged to home, and 58 patients entered the analysis. Data analysis was conducted by a researcher who was blind to the data.

\section{Results}

Two patients were discharged from the hospital in the control group, and we lost them in the follow-up. In the intervention group, 17 (56.7\%) subjects were female and $13(43.3 \%)$ subjects were male. In the control group, 14 (46.7\%) subjects were female and $14(46.7 \%)$ subjects

Table 1. Comparison of the Severity of Diaper Dermatitis Based on Mother and Child Age and Child Weight

\begin{tabular}{lccccc}
\hline \multirow{2}{*}{ Variable } & $\begin{array}{c}\text { Intervention group } \\
(\mathbf{n}=\mathbf{3 0}) \\
\text { Mean } \pm \text { SD }\end{array}$ & $\begin{array}{c}\text { Control group } \\
(\mathbf{n = 2 8 )} \\
\text { Mean } \pm \text { SD }\end{array}$ & & F & $\begin{array}{c}\boldsymbol{P} \\
\text { Value }^{\mathrm{a}}\end{array}$ \\
\hline Child age (day) & $90.96 \pm 140.20$ & $55.58 \pm 146.73$ & 1.137 & 0.32 \\
\hline Mothers age $(\mathrm{y})$ & $28.53 \pm 6.36$ & $26.30 \pm 6.29$ & 1.02 & 0.36 \\
\hline Child weight $(\mathrm{g})$ & $4272.93 \pm 2261.78$ & $3610.17 \pm 2003.56$ & 0.56 & 0.57 \\
\hline Child gender & $1.366 \pm 0.49$ & $1.50 \pm 0.50$ & 2.07 & 0.31 \\
\hline a Independent $t$ test. & & & &
\end{tabular}

Table 2. Comparison of the Severity of Diaper Dermatitis on 1st, 3rd and 6th Days of Intervention in the Control and Intervention Groups

\begin{tabular}{|c|c|c|c|c|}
\hline \multirow{3}{*}{$\begin{array}{l}\text { Severity of } \\
\text { Dermatitis }\end{array}$} & \multicolumn{2}{|c|}{ Groups } & \multirow{3}{*}{$F$} & \multirow{3}{*}{$P$ Value $^{a}$} \\
\hline & $\begin{array}{l}\text { Intervention group } \\
\qquad(\mathbf{n}=\mathbf{3 0})\end{array}$ & $\begin{array}{c}\text { Control group } \\
(\mathbf{n}=\mathbf{2 8})\end{array}$ & & \\
\hline & Mean \pm SD & Mean \pm SD & & \\
\hline First day & $1.23 \pm 1.13$ & $0.8 \pm 0.88$ & 0.49 & 0.26 \\
\hline Third day & $086 \pm 086$ & $0.43 \pm 0.62$ & 0.29 & 0.82 \\
\hline Sixth day & $0.53 \pm 1.04$ & $0.16 \pm 0.37$ & 0.23 & 0.16 \\
\hline$P$ value ${ }^{b}$ & 0.001 & 0.001 & & \\
\hline
\end{tabular}

a Independent $t$ test.

${ }^{\mathrm{b}}$ Repeated measure test.

Table 3. The Results of ANOVA With Repeated-Measures of the Severity of Diaper Dermatitis in the Intervention Group

\begin{tabular}{lccccc}
\hline \multicolumn{7}{c}{ Tests of Within-Subjects Effects } \\
\hline Source & $\begin{array}{c}\text { Type III Sum of } \\
\text { Squares }\end{array}$ & df & $\begin{array}{c}\text { Mean } \\
\text { Square }\end{array}$ & F & P Value \\
\hline Time & 13.378 & 1.486 & 9.006 & 20.113 & 0.000 \\
\hline Time * group & 0.44 & 1.486 & 0.030 & 0.067 & 0.886 \\
\hline Error (Time) & 38.578 & 86.160 & 0.448 & & \\
\hline
\end{tabular}

Table 4. The Results of ANOVA With Repeated-Measures of the Severity of Diaper Dermatitis in the Intervention and Control Groups

\begin{tabular}{cccccc}
\hline \multicolumn{5}{c}{ Tests of Between-Subjects Effects } \\
\hline Source & $\begin{array}{c}\text { Type III Sum of } \\
\text { Squares }\end{array}$ & df & $\begin{array}{c}\text { Mean } \\
\text { Square }\end{array}$ & F & P Value \\
\hline Intercept & 81.339 & 1 & 81.339 & 52.386 & 0.000 \\
\hline Group & 7.606 & 1 & 7.606 & 4.898 & 0.031 \\
\hline Error & 90.056 & 58 & 1.553 & & \\
\hline
\end{tabular}

were male. The children's mean age in the control and intervention groups was $55.58 \pm 74.22$ and $90.20 \pm 140.20$ days, respectively. The mothers' mean age in the intervention and control groups was $28.53 \pm 6.36$ and $26.30 \pm 6.29$ years, respectively. The results of independent $t$ test indicated no significant differences between the two groups in terms of the neonatal age, gender, weight, and maternal age $(P>0.05)$ (Table 1$)$.

The results of repeated-measures ANOVA indicated that the severity of dermatitis significantly improved in both groups on the sixth day compared to the first day (Table 2).

The Greenhouse-Geisser correction was used for repeated-measures ANOVA. In tests of within-subjects effects based on $\mathrm{F}(1.48,86.160)=20.113$ and $P<0.001$, the severity of dermatitis was statistically significant in the intervention group, and it decreased in children over time. The results also showed that the effect of time is not statistically significant on the studied groups, and passing time had no effect on reducing the severity of dermatitis in children $(\mathrm{F}(1.486,86.160)=0.067$ and $P=0.886)$ (Table 3).

In addition, tests of between-subjects effects showed that the mean severity of dermatitis score was statistically significant between the intervention and control groups; 
as a result, using aloe vera ointment reduced the severity of dermatitis in children in the intervention group over time $(\mathrm{F}(1,58)=4.898$ and $P=0.031)$ (Table 4$)$.

A significant reduction was seen in the mean severity of the dermatitis score in the intervention group over time (Figure 2). No adverse effects were noted in the two groups during and after the intervention.

\section{Discussion}

This study compared the effects of aloe vera ointment with routine treatment on the severity of DD in children. The results indicated that the severity of dermatitis improved in both groups within six days following treatment, but neither group was superior to the other. Herbal products have been used mainly in traditional medicine. Lately, researchers have studied the anti-inflammatory effects of herbal remedies in treating several inflammatory diseases, such as DD. Al-Waili investigated the healing impact of a mixture of honey, olive oil, and beeswax on 12 children with DD; the children were treated with cream four times a day for seven days. Their results showed that the severity of DD was significantly decreased in most patients after seven days (15). In another study, the positive therapeutic effect of olive oil was confirmed on 173 children with DD (18). The anti-inflammatory effect of aloe vera is due to the presence of thromboxane and cyclooxygenase derivatives in it (13). Akbari et al showed that aloe vera could positively treat DD (12). The positive effect of aloe vera products on DD has been confirmed in several recent studies $(4,8,10)$. However, Panahi et al compared the effect of aloe vera ointment with calendula ointment on the severity of $\mathrm{DD}$ and reported that dermatitis improved more slowly with aloe vera ointment than calendula ointment (11), that was inconsistent with our findings. This might be due to the more antimicrobial and anti-inflammatory effects of calendula compared to aloe vera. A recent study compared the effects of aloe vera and chamomile ointment on the severity of $\mathrm{DD}$, and found that they effectively improved DD, but neither was superior to the other (19). Vardy et al studied the effect of aloe vera extract on patients with seborrheic dermatitis, and concluded that inflammation and cutaneous scaling significantly reduced in patients treated with aloe vera (20). In our study, DD improvement from baseline was observed in both groups, and aloe vera ointment had a higher therapeutic effect than the routine ointment. This beneficial effect could be associated with the well-known anti-inflammatory and antimicrobial properties of aloe vera ointment $(2,11,13,21)$.

According to the existing literature, some of the researchers used aloe vera gel in their studies (22-24). We used aloe vera ointment instead of aloe vera gel because the gel form is usually water-based but ointment is oilbased. Ointments are occlusive, indicating when used, they leave a layer of oil on the skin's surface. This layer of oil traps moisture in to prevent wounds from drying out.

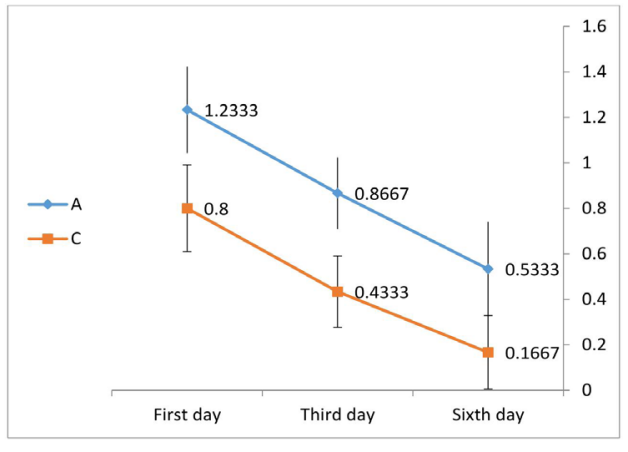

$A=$ Aloe Vera group

$\mathrm{C}=$ Control group

Figure 2. Diagram of the Mean Severity of Dermatitis Scores in the Intervention Group and its Comparison with the Control Group.

Gels also absorb well and only leave behind a thin layer from the thickening agent. Ointments are more suitable for dry skin, but gels are good for oily skin.

The main limitation of current study was the lack of a precise range of severity of DD in the Al-Waili scale. We recommend using a newly developed precise scale in a similar study to investigate the effect of aloe vera in the severity of DD more precisely. In this study, the samples were selected from children with different diets and diaper brands, which could affect our results. Individual differences among patients was another limitation of our study. In addition, ignoring children's gender differences, their length of hospital stay, and failure to check zinc levels were other limitations that could affect the results. Researchers in future studies might consider gender differences and check zinc levels. We also suggest that patients with fungal DD be included in future research to assess the impact of aloe vera extracts on fungal infection. Furthermore, other herbal treatments could be used and compared with aloe vera ointment.

\section{Conclusion}

In the current research, the emphasis was on the antiinflammatory properties of aloe vera. Herbal products cause fewer side effects than chemical products, and they seem to have significant pharmacological effects on the skin (9). Thus, aloe vera ointment appears to help treat $\mathrm{DD}$, and with further studies, may emerge as a possible therapeutic option.

\section{Conflict of Interest Disclosures}

The authors declare that they have no conflict of interests.

\section{Acknowledgments}

This article is part of a master's thesis on pediatric nursing (Registration no. 667). The authors would like to thank the research deputy of the Tabriz University of Medical Science, Iran for financially supporting the project. They would also show their profound gratitude toward the Tabriz pediatric hospital personnel and parents of the hospitalized children participating in the research for their cooperation. They also wish to express their appreciation 
to Mariam Angelica Parizad for reviewing the manuscript and writing assistance.

\section{Ethical Statement}

The study protocol was approved by the Research Ethics Committee of Tabriz University of Medical Sciences (IR.TBZMED. REC.1395.872) and registered in the Iranian Clinical Trial Registration System (identifier: IRCT2016082813691N10; https:// www.irct.ir/trial/13484). The parents of all children signed a written informed consent letter, and they were assured of the confidentiality of their information. The parents of all patients were free to leave the study at any time.

\section{Authors' Contributions}

All authors have materially participated in the research and manuscript preparation. Study concept and design: SGB, NP; analysis and interpretation of data: $\mathrm{SGB}, \mathrm{NP}$, and $\mathrm{SH}$; drafting of the manuscript: NP, SGB, and FS; critical revision of the manuscript for important intellectual content: NP, SGB, SH, FS; statistical analysis: NP, VA; final manuscript review and approval: NP, SGB, SH, FS, VA.

\section{Funding/Support}

The current study was funded by Tabriz University of Medical Sciences.

\section{Informed Consent}

The participants were fully informed about the purpose of the study. Each participant provided a written consent prior to participation. The voluntary nature of participation was explained to all participants and all participants were free to leave the study at any time. They were also assured about the privacy and confidentiality of their information.

\section{References}

1. Oranje AP, Bonifazi E, Honig PJ, Yan AC. Napkin dermatitis. In: Harper's Textbook of Pediatric Dermatology. Hoboken, NJ: John Wiley \& Sons Ltd; 2019. p. 265-78.

2. Sharifi-Heris Z, Amiri Farahani L, Hasanpoor-Azghadi SB. A review study of diaper rash dermatitis treatments. J Client Centered Nurs Care. 2018;4(1):1-12. doi: 10.32598/ jccnc.4.1.1.

3. Ghanipour Badelbuu S, Javadzadeh Y, Jabraeili M, Heidari S, Etebar Matanag N, Arshadi M. Effects of Aloe vera gel versus chamomile ointment on extent of diaper dermatitis in children: a double-blind randomized controlled trial. Int J Pediatr. 2019;7(5):9461-9. doi: 10.22038/ijp.2018.28232.2443.

4. Blume-Peytavi U, Hauser M, Lünnemann L, Stamatas GN, Kottner J, Garcia Bartels N. Prevention of diaper dermatitis in infants--a literature review. Pediatr Dermatol. 2014;31(4):41329. doi: 10.1111/pde.12348.

5. Cohen B. Differential diagnosis of diaper dermatitis. Clin Pediatr (Phila). 2017;56(5_Suppl):16S-22S. doi: 10.1177/0009922817706982.

6. Stamatas GN, Tierney NK. Diaper dermatitis: etiology, manifestations, prevention, and management. Pediatr Dermatol. 2014;31(1):1-7. doi: 10.1111/pde.12245.

7. Sajjad A, Subhani Sajjad S. Aloe vera: an ancient herb for modern dentistry - a literature review. J Dent Surg. 2014;2014:210463. doi: 10.1155/2014/210463.

8. Klunk C, Domingues E, Wiss K. An update on diaper dermatitis. Clin Dermatol. 2014;32(4):477-87. doi: 10.1016/j. clindermatol.2014.02.003.

9. Tabassum N, Hamdani M. Plants used to treat skin diseases. Pharmacogn Rev. 2014;8(15):52-60. doi: 10.4103/09737847.125531.
10. Hugill K. Revisiting infant nappy dermatitis: causes and preventive care. Br J Midwifery. 2017;25(3):150-4. doi: 10.12968/bjom.2017.25.3.150

11. Panahi Y, Sharif MR, Sharif A, Beiraghdar F, Zahiri Z, Amirchoopani G, et al. A randomized comparative trial on the therapeutic efficacy of topical aloevera and Calendula officinalis on diaper dermatitis in children. ScientificWorldjournal. 2012;2012:810234. doi: 10.1100/2012/810234.

12. Akaberi M, Sobhani Z, Javadi B, Sahebkar A, Emami SA. Therapeutic effects of Aloe spp. in traditional and modern medicine: a review. Biomed Pharmacother. 2016;84:759-72. doi: 10.1016/j.biopha.2016.09.096.

13. Heng HC, Zulfakar MH, Ng PY. Pharmaceutical applications of Aloe vera. Indones J Pharm. 2018;29(3):101-16. doi: 10.14499/indonesianjpharm29iss3pp101.

14. Lebsing S, Chaiyarit J, Techasatian L. Diaper rashes can indicate systemic conditions other than diaper dermatitis. BMC Dermatol. 2020;20(1):7. doi: 10.1186/s12895-02000104-z.

15. Al-Waili NS. Clinical and mycological benefits of topical application of honey, olive oil and beeswax in diaper dermatitis. Clin Microbiol Infect. 2005;11(2):160-3. doi: 10.1111/j.1469-0691.2004.01013.x.

16. Noonan C, Quigley S, Curley MA. Skin integrity in hospitalized infants and children: a prevalence survey. J Pediatr Nurs. 2006;21(6):445-53. doi: 10.1016/j.pedn.2006.07.002

17. Afshari Z, Jabraeili M, Asaddollahi M, Ghojazadeh M, Javadzadeh Y. Comparison of the effects of chamomile and calendula ointments on diaper rash. Evidence Based Care. 2015;5(2):49-56. doi: 10.22038/ebcj.2015.4544.

18. Kiechl-Kohlendorfer $U$, Berger $C$, Inzinger $R$. The effect of daily treatment with an olive oil/lanolin emollient on skin integrity in preterm infants: a randomized controlled trial. Pediatr Dermatol. 2008;25(2):174-8. doi: 10.1111/j.15251470.2008.00627.x.

19. Badelbuu SG, Javadzadeh Y, Jabraeili M, Heidari S, Bostanabad MA. Evaluation of the effect of Aloe vera ointment with chamomile ointment on severity of children's diaper dermatitis: a randomized, double-blind clinical trial. World Family Medicine Journal: Incorporating the Middle East Journal of Family Medicine Middle East J Fam Med. 2018;99(5897):1-5. doi: 10.5742/mewfm.2018.93304.

20. Vardy D, Cohen A, Tchetov T, Medvedovsky E, Biton A. A double-blind, placebo-controlled trial of an Aloe vera (A. barbadensis) emulsion in the treatment of seborrheic dermatitis. J Dermatolog Treat. 1999;10(1):7-11. doi: 10.3109/09546639909055904.

21. Zagórska-Dziok M, Furman-Toczek D, Dudra-Jastrzębska M, Zygo K, Stanisławek A, Kapka-Skrzypczak L. Evaluation of clinical effectiveness of Aloe vera-a review. Journal of Pre-Clinical and Clinical Research. 2017;11(1):86-93. doi: 10.26444/jpccr/74577.

22. Najafian Y, Khorasani ZM, Najafi MN, Hamedi SS, Mahjour M, Feyzabadi Z. Efficacy of Aloe vera/Plantago major gel in diabetic foot ulcer: a randomized double-blind clinical trial. Curr Drug Discov Technol. 2019;16(2):223-31. doi: 10.2174/ 1570163815666180115093007.

23. Khan AW, Kotta S, Ansari SH, Sharma RK, Kumar A, Ali J. Formulation development, optimization and evaluation of Aloe vera gel for wound healing. Pharmacogn Mag. 2013;9(Suppl 1):S6-S10. doi: 10.4103/0973-1296.117849.

24. Molazem Z, Mohseni F, Younesi M, Keshavarzi S. Aloe vera gel and cesarean wound healing; a randomized controlled clinical trial. Glob J Health Sci. 2014;7(1):203-9. doi: 10.5539/gjhs.v7n1p203. 